\title{
Influencia de la adición de cobre y de bronce sobre las propiedades de los aceros inoxidables austeníticos sinterizados ${ }^{\left({ }^{\circ}\right)}$
}

\author{
F. Velasco ${ }^{(*)}$, N. Antón $\left(^{(*)}\right.$, N. Candela ${ }^{(* *)}$ y J.M. Torralba $(* *)$ \\ Resumen Partiendo de los aceros inoxidables AISI 316L y 304L a los que se añadió cobre y bronce en \\ diferentes porcentajes hasta el $20 \%$ en peso, se estudia el efecto que la cantidad de aleante y la \\ temperatura de sinterización tienen sobre las propiedades físicas y mecánicas y sobre la \\ microestructura de los aceros inoxidables sinterizados. La aleación con cobre y con bronce aumenta \\ la densidad de los aceros sinterizados para las dos temperaturas de sinterización utilizadas. El cobre y \\ el bronce provocan la aparición de fase líquida (transitoria o permanente) que activa el proceso de \\ sinterización. La resistencia a la tracción del acero inoxidable experimenta un fuerte incremento para \\ los contenidos más elevados de aleante. Además, la resistencia a la tracción alcanza mayores valores \\ para la aleación con bronce que con cobre.
}

Palabras clave: Acero inoxidable. Aleación. Pulvimetalurgia.

\section{Copper and bronze influence on sintered austenitic stainless steels properties}

\begin{abstract}
The effect that, on AISI 316L and 304L stainless steels alloyed with copper and bronze in different percentages up to a maximun of $20 \% \mathrm{wt}$, produce both the alloying content and the sintering temperature over physical and mechanical properties and over the microstructure of sintered stainless steels are studied. Alloying with copper and bronze improves the density of sintered steels at the two sintering temperatures used. Copper and bronze promote liquid phase sintering (transitory or permanent), that activates sintering process. Tensile strength of stainless steel is highly improved for higher alloying contents. Moreover, tensile strength presents greater values alloying with bronze than with copper.
\end{abstract}

Keywords: Stainless steel. Alloying. Powder metallurgy.

\section{INTRODUCCIÓN}

Los aceros inoxidables tienen mucha aplicación en pulvimetalurgia debido a la buena combinación de propiedades mecánicas y resistencia a la corrosión (1). Además, las técnicas pulvimetalúrgicas proporcionan otras ventajas, como el ahorro de materias primas, el excelente control dimensional y un buen acabado. Entre las aplicaciones típicas de los aceros inoxidables sinterizados se incluyen los

(•) Trabajo recibido el día 15 de febrero de 1996.

(*) Universidad Jaime I. Dept. de Tecnología. Campus Penyeta Roja. 12071-Castellón (España).

(**) E.T.S. de Ingenieros de Minas. Dept. de Materiales. C/ Ríos Rosas, 21. 28003-Madrid (España). filtros, los anillos ABS de los automóviles y diversos componentes para la industria alimentaria.

La fase más importante del proceso cuando se persigue obtener las propiedades deseadas en los aceros inoxidables, es la sinterización. Las condiciones óptimas de sinterización son las siguientes (2): elevada temperatura de sinterización, bajo punto de rocío en la atmósfera, bajos contenidos de carbono, de oxígeno y de nitrógeno en el acero y enfriamiento rápido.

Sin embargo, los aceros inoxidables sinterizados tienen peor resistencia a la corrosión que los convencionales debido a la porosidad interconectada. Aunque el efecto de la densidad de sinterización no se conoce bien (3 y 4), la resistencia a la corrosión 
se ha intentado mejorar mediante la aleación de los aceros inoxidables con diversos elementos, tales como cobre, estaño o silicio (5-8), y se ha estudiado la posibilidad de infiltrarlos con cobre y con bronce (9).

En este trabajo, se estudia la posibilidad de alear los aceros inoxidables AISI 316L y 304L con cobre y con bronce siguiendo las técnicas convencionales de pulvimetalurgia. Ambos aleantes se añadieron en diferentes porcentajes hasta un máximo del $20 \%$ en peso, y se investiga la influencia que, tanto la cantidad de aleante como la temperatura de sinterización, tienen sobre las propiedades de los aceros inoxidables sinterizados. El cobre y el bronce provocan la aparición de fases líquidas que activan el proceso de sinterización y que influyen de manera importante sobre las propiedades físicas y mecánicas de los aceros, así como sobre su microestructura.

\section{PROCEDIMIENTO EXPERIMENTAL}

El plan experimental se estableció de forma que se pudiera determinar la influencia, tanto de la cantidad de aleante como de la temperatura de sinterización, sobre las propiedades físicas (densidades en verde y de sinterización, variación dimensional) y las propiedades mecánicas (resistencia a la tracción y alargamiento) de los aceros inoxidables pulvimetalúrgicos aleados con cobre y con bronce. Para ello, se emplearon, como polvos base, los aceros inoxidables austeníticos AISI 316L y 304L, y como aleantes, polvos de cobre electrolítico y bronce prealeado (atomizado), en cuatro porcentajes: 4, 8, 15 y $20 \%$ (en masa). Las propiedades de los aceros aleados se compararon con las de los aceros inoxidables base utilizados. La tabla I muestra las características, tanto de los polvos base como de los polvos de cobre y de bronce.

La mezcla de materiales se realizó en seco, en un mezclador de laboratorio, durante $15 \mathrm{~min}$, hasta asegurar la homogeneidad de la mezcla. Posteriormente, el polvo se compactó uniaxialmente en matriz flotante a $700 \mathrm{MPa}$, con la forma de la probeta de tracción normalizada (MPIF Standard 10, ASTM E-69) utilizada para productos sinterizados. Los compactos en verde se controlaron dimensionalmente y se midió la densidad, también en verde, de los distintos aceros.

La sinterización se realizó en vacío a dos temperaturas diferentes, 1.150 y $1.250{ }^{\circ} \mathrm{C}$, durante 15 min. La temperatura inferior fue elegida con el fin de estudiar la sinterización a temperaturas próximas a las empleadas industrialmente, mientras que la superior se eligió partiendo de la temperatura óptima de sinterización de estos aceros inoxidables $\left(1.330^{\circ} \mathrm{C}\right)(10)$ y suponiendo una cierta activación
Tabla I.- Características de los distintos polvos empleados en la investigación

TABLE I.- Characteristics of the different powders used in this investigation

\begin{tabular}{|c|c|}
\hline $316 \mathrm{LHC}$ & $\begin{array}{l}\text { Densidad: } 8,0 \mathrm{~g} / \mathrm{cm}^{3} \\
\text { Composición química, \%: } 0,02 \mathrm{C} ; 13,55 \mathrm{Ni} \text {; } \\
16,1 \mathrm{Cr} \text {; } 0,87 \mathrm{Si} ; 2,24 \mathrm{Mo} ; 0,02 \mathrm{Cu} \\
\text { Granulometría: } 99,8 \%<150 \mu \mathrm{m} \\
\text { Densidad aparente: } 2,95 \mathrm{~g} / \mathrm{cm}^{3} \\
\text { Velocidad de flujo: } 28,3 \mathrm{~s} / 50 \mathrm{~g} \\
\text { Suministrador: Coldstream (Bélgica) }\end{array}$ \\
\hline $304 \mathrm{~L}$ & $\begin{array}{l}\text { Densidad: } 7,9 \mathrm{~g} / \mathrm{cm}^{3} \\
\text { Composición química, \%: 0,009 C; } 11,4 \mathrm{Ni} \text {; } \\
18,15 \mathrm{Cr} ; 0,78 \mathrm{Si} \\
\text { Granulometría: } 99,5 \%<150 \mu \mathrm{m} \\
\text { Densidad aparente: } 2,87 \mathrm{~g} / \mathrm{cm}^{3} \\
\text { Velocidad de flujo: } 27,8 \mathrm{~s} / 50 \mathrm{~g} \\
\text { Suministrador: Coldstream (Bélgica) }\end{array}$ \\
\hline Cobre & $\begin{array}{l}\text { Fabricado mediante electrólisis } \\
\text { Composición química, } \%: \geq 99,7 \mathrm{Cu} ; \leq 0,17 \mathrm{O} \\
\text { Granulometría: } 99,8 \%<80 \mu \mathrm{m} ; \\
85-95 \%<40 \mu \mathrm{m} \\
\text { Suministrador: Norddeutsche Affinerie } \\
\text { (Alemania) }\end{array}$ \\
\hline Bronce & $\begin{array}{l}\text { Polvos prealeados } \\
\text { Composición química, } \% \text { : } 90 \mathrm{Cu}-10 \mathrm{Sn} \\
\text { Granulometría: } 90 \%<50 \mu \mathrm{m} ; 99 \%<75 \mu \mathrm{m}\end{array}$ \\
\hline
\end{tabular}

de la sinterización gracias a la fase líquida a que da lugar la aleación con cobre y con bronce. Se midió la variación dimensional existente durante el proceso de sinterización (evaluada como variación de la longitud de la probeta sinterizada con respecto a la de la probeta en verde), y se controlaron la densidad de sinterización y las propiedades mecánicas (resistencia a la tracción y alargamiento), tanto de los aceros aleados como de los aceros inoxidables base.

La densidad de los distintos materiales se determinó mediante el método de Arquímedes en una balanza Mettler de $0,0001 \mathrm{~g}$ de precisión. El cálculo de la densidad se realizó aplicando la siguiente fórmula:

$$
\rho_{\mathrm{s}}=\frac{M}{P_{\mathrm{tot}}-\frac{P_{\mathrm{tot}}-P_{\mathrm{a}}}{\rho_{\text {sell }}}}
$$

siendo $\rho_{\mathrm{s}}$ la densidad del producto sinterizado, $M$ el peso del producto sinterizado, $P_{\text {tot }}$ el peso del material con sellante, $P_{\mathrm{a}}$ el peso del material sumergido, y $\rho_{\text {sell }}$ la densidad del sellante.

También se realizó un amplio estudio microestructural mediante microscopía óptica convencional. La preparación metalográfica de los distintos 
aceros se realizó por métodos convencionales, utilizándose, en la fase de pulido final, alúmina $(5 \mu \mathrm{m})$ en suspensión acuosa y polvo de diamante $(1 \mu \mathrm{m})$. Como reactivos de ataque se emplearon:

a) $45 \mathrm{~cm}^{3} \mathrm{HCl}+15 \mathrm{~cm}^{3} \mathrm{HNO}_{3}+20 \mathrm{~cm}^{3}$ etanol.

b) $44 \mathrm{~cm}^{3} \mathrm{H}_{2} \mathrm{O}+5 \mathrm{~cm}^{3} \mathrm{HNO}_{3}+1 \mathrm{~cm}^{3} \mathrm{HF}(48$ $\%)$, en caliente.

\section{RESULTADOS}

La figura 1 muestra la densidad en verde de los aceros inoxidables $316 \mathrm{~L}$ y $304 \mathrm{~L}$ mezclados con cobre y con bronce. Como era de esperar, en ambos casos, esta propiedad aumenta con el contenido de aleante, por un lado al incrementarse la compresibilidad de los polvos, y por otro, debido a la inclusión de materiales (cobre y bronce) de mayor densidad que el acero inoxidable.

La figura 2 muestra la densidad de sinterización del acero inoxidable $316 \mathrm{~L}$ aleado. En la mayoría de los casos, la densidad del acero inoxidable mejora al ser aleado, lo que es lógico, si a los motivos ya reseñados se añade el hecho de realizar la sinterización en fase líquida que activa el proceso.

En la figura 3 se presentan los resultados obtenidos para la variación dimensional del acero inoxidable $304 \mathrm{~L}$. Se puede apreciar que se produce un hinchamiento de los aceros inoxidables para contenidos intermedios de cobre (a ambas temperaturas de sinterización) y de bronce (al sinterizar a $\left.1.250{ }^{\circ} \mathrm{C}\right)$.

Como se observa en la figura 4 , correspondiente al acero $316 \mathrm{~L}$, la resistencia a la tracción de los ace-

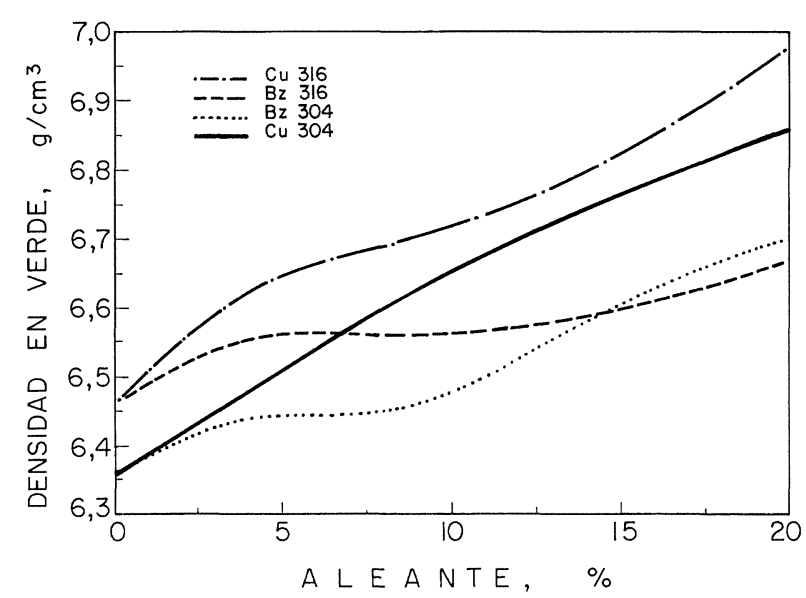

FIG. 1.- Densidad en verde de los aceros inoxidables 316L y 304L aleados con cobre y con bronce.

FIG. 1.- Green density of $316 L$ and $304 L$ stainless steels alloyed with copper and bronze.

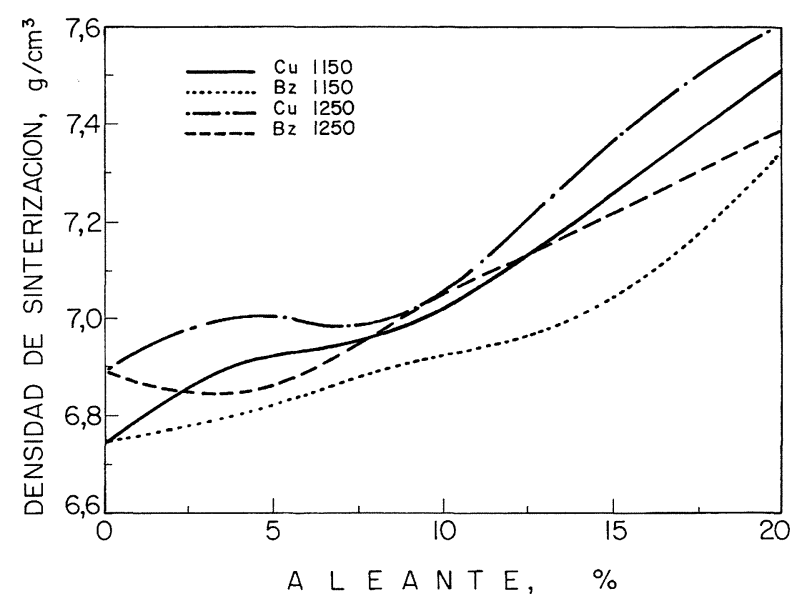

FIg. 2.- Densidad de sinterización del acero inoxidable $316 \mathrm{~L}$ aleado con cobre y con bronce.

FIG. 2.- Sintering density of $316 L$ stainless steel alloyed with copper and bronze.

roś inoxidables aleados con cobre y con bronce es superior a la de los aceros inoxidables base en el caso de altos contenidos de aleante (15 y $20 \%$ ), debido a la presencia de fase líquida permanente durante la sinterización. Sin embargo, para los contenidos bajos tanto de cobre como de bronce, y para ambas temperaturas de sinterización, la resistencia a la tracción no varía sustancialmente respecto a los aceros inoxidables sin alear.

En la figura 5, corrrespondiente al acero 304L, se representan los valores de alargamiento de los distintos aceros. Se puede observar que en esta propiedad no influye mucho la aleación.

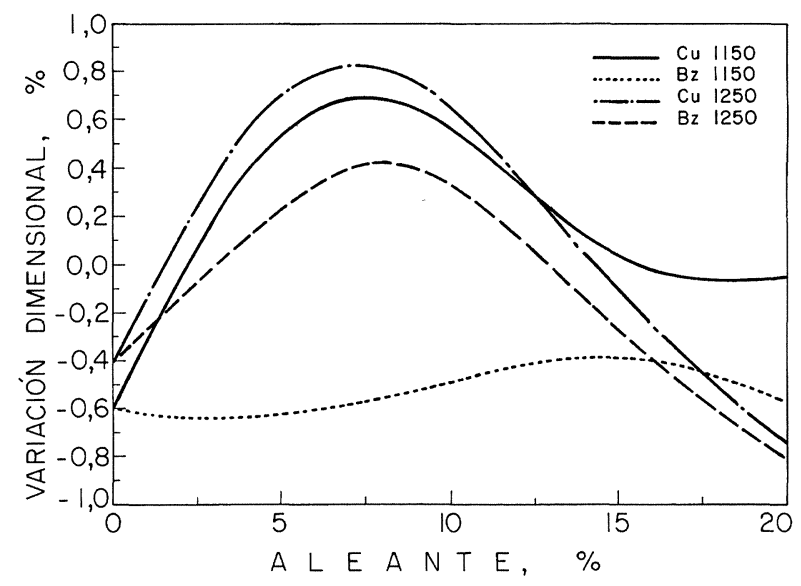

FIG. 3.- Variación dimensional del acero inoxidable 304L aleado con cobre y con bronce.

FIG. 3.- Dimensional change of 304L stainless steel alloyed with copper and bronze. 


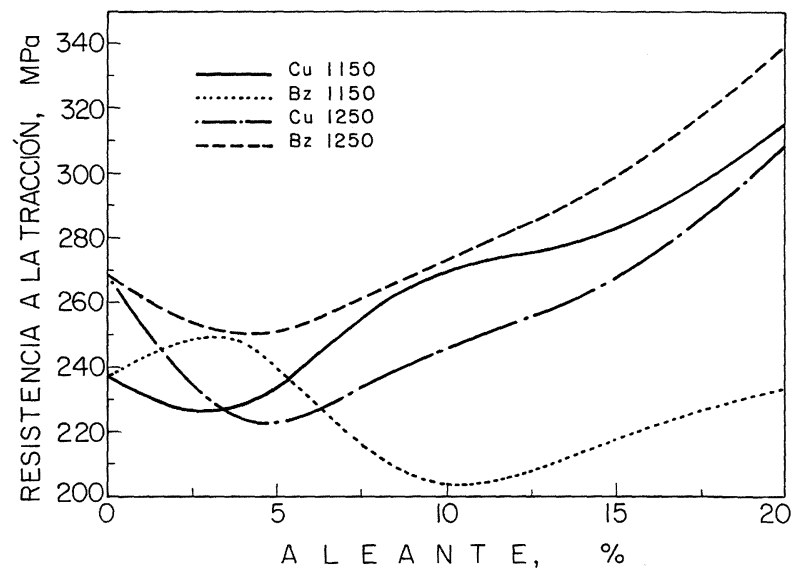

FIG. 4.- Resistencia a la tracción del acero inoxidable $316 \mathrm{~L}$ aleado con cobre y con bronce.

FIG. 4.- Tensile strength of $316 \mathrm{~L}$ stainless steel alloyed with copper and bronze.

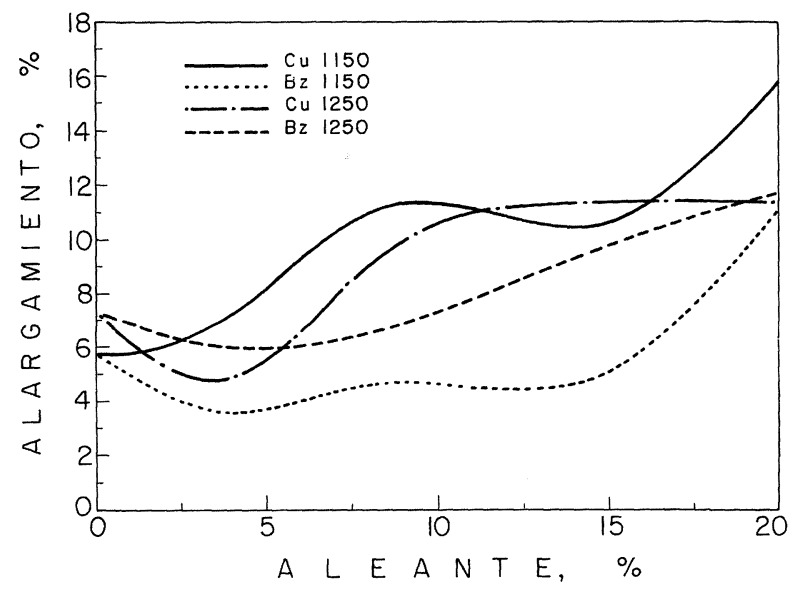

FIG. 5.- Alargamiento del acero inoxidable 304L aleado con cobre y con bronce.

FIG. 5.- Elongation of 304L stainless steel alloyed with copper and bronze.

\section{ESTUDIO MICROESTRUCTURAL}

El estudio metalográfico muestra microestructuras similares para la aleación con bronce o con cobre. Al sinterizar a $1.150{ }^{\circ} \mathrm{C}$, se puede observar que al alear el acero un $4 \%$, el aleante difunde totalmente (Fig. 6, correspondiente al acero 304L con $4 \% \mathrm{Cu}$ ). Así, en los aceros inoxidables sin alear y aleados con un $4 \%$ de cobre o de bronce, lo único que se aprecia en las microestructuras es la porosidad del material y la estructura austenítica del acero. $\mathrm{Al}$ añadir un $8 \%$, el aleante difunde casi en su totalidad, aunque se empiezan a mostrar áreas de fase líquida (Fig. 7, acero 316L con $8 \% \mathrm{Cu}$ ). En el caso de la sinterización a $1.250{ }^{\circ} \mathrm{C}$, la mayor tempe-

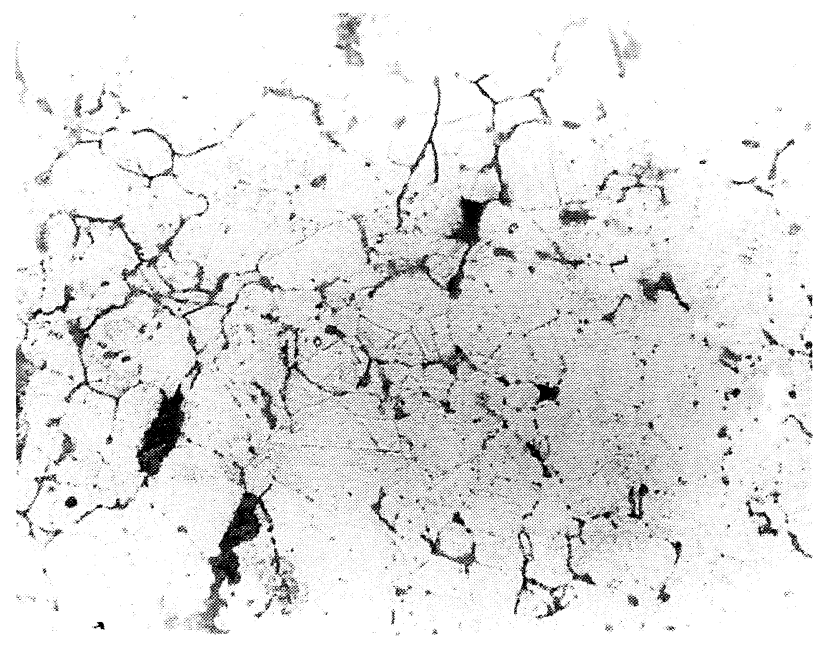

FIG. 6.- Microestructura del acero inoxidable 304L aleado con $4 \%$ de cobre sinterizado a 1.150 ${ }^{\circ} \mathrm{C}$. Ataque: $\mathrm{HCl}+\mathrm{HNO}_{3}+$ etanol. $\times 125$.

FIG. 6.- Microstructure of 304L stainless steel alloyed with $4 \%$ copper sintered at $1,150{ }^{\circ} \mathrm{C}$. Etching: $\mathrm{HCl}+\mathrm{HNO}_{3}+$ ethanol. $\times 125$.

ratura activa los fenómenos de difusión. A nivel microestructural, se obtienen estructuras similares a las obtenidas al sinterizar a $1.150{ }^{\circ} \mathrm{C}$ para contenidos de aleante del $4 \%$ (no aparece cobre ni bronce libre). Sin embargo, en el caso del $8 \%$ de aleante, todo el cobre y el bronce han desaparecido por difusión al sinterizar a $1.250{ }^{\circ} \mathrm{C}$ (Fig. 8, acero 316L con $8 \%$ de bronce).

Finalmente, el empleo de altos contenidos de aleante produce grandes áreas de fase líquida, tanto

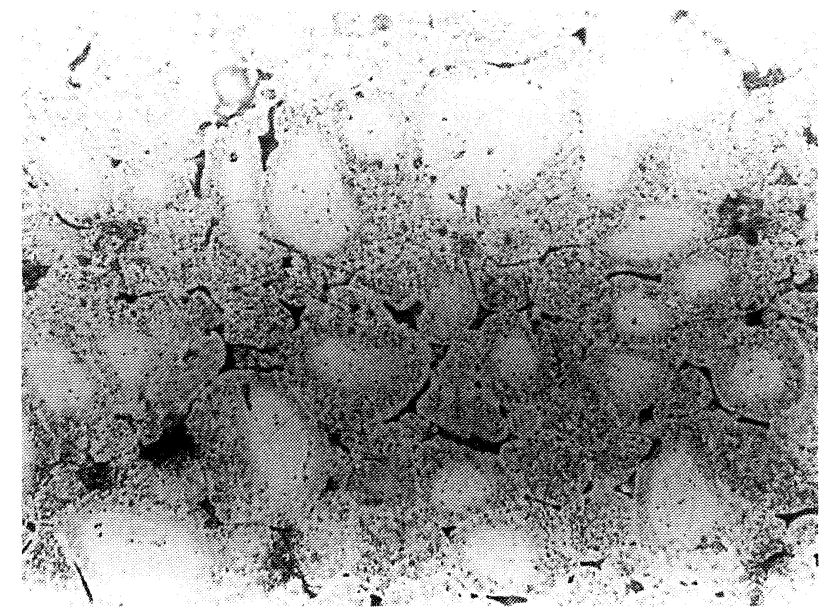

FIG. 7.- Microestructura del acero inoxidable $316 \mathrm{~L}$ aleado con $8 \%$ de cobre sinterizado a 1.150 ${ }^{\circ} \mathrm{C}$. Ataque: $\mathrm{HCl}+\mathrm{HNO}_{3}+$ etanol. $\times 165$.

FIG. 7.- Microstructure of $316 \mathrm{~L}$ stainless steel alloyed with $8 \%$ copper sintered at $1,150{ }^{\circ} \mathrm{C}$. Etching: $\mathrm{HCl}+\mathrm{HNO}_{3}+$ ethanol. $\times 165$. 


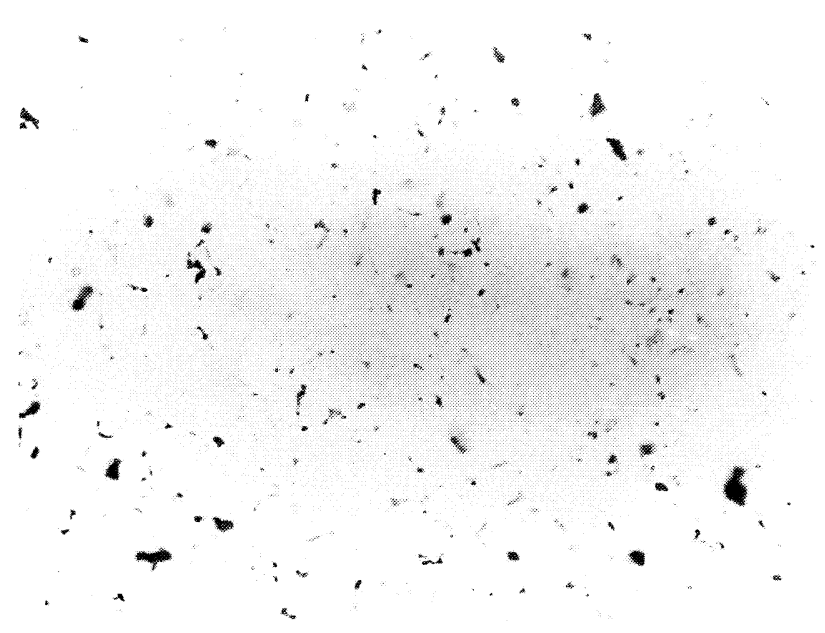

FIG. 8.- Microestructura del acero inoxidable 304L aleado con $8 \%$ de bronce sinterizado a 1.250

${ }^{\circ} \mathrm{C}$. Sin ataque. $\times 50$.

FIG. 8.- Microstructure of 304L stainless steel alloyed with $8 \%$ bronze sintered at $1,250{ }^{\circ} \mathrm{C}$. Without etching. $\times 50$.

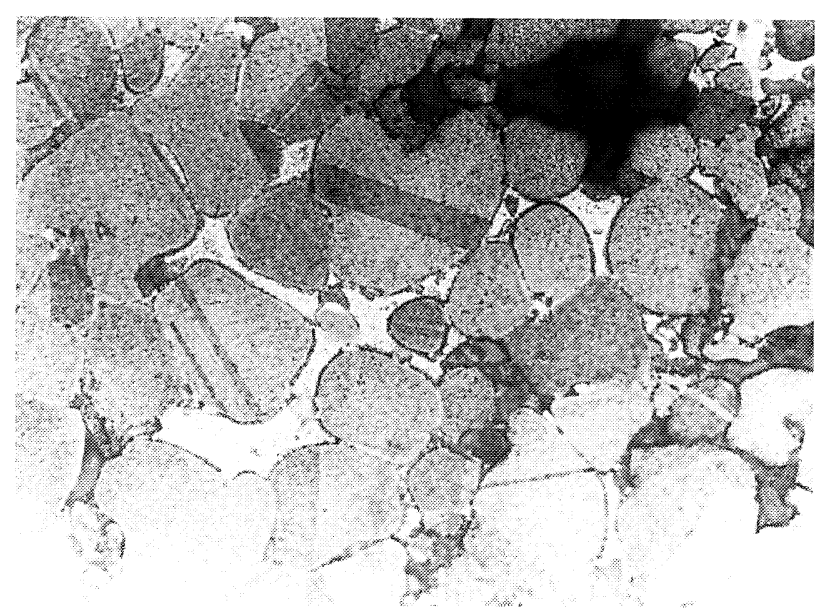

FIG. 9.- Microestructura del acero inoxidable 304L aleado con $15 \%$ de bronce sinterizado a $1.250{ }^{\circ} \mathrm{C}$. Ataque: $\mathrm{H}_{2} \mathrm{O}+\mathrm{HNO}_{3}+\mathrm{HF}$ en caliente. $\times 125$.

FIG. 9.- Microstructure of $304 \mathrm{~L}$ stainless steel alloyed with $15 \%$ bronze sintered at $1,150{ }^{\circ} \mathrm{C}$. Etching: $\mathrm{H}_{2} \mathrm{O}+\mathrm{HNO}_{3}+\mathrm{HF}$, hot dipping. $\times 125$.

al sinterizar a 1.150 como a $1.250{ }^{\circ} \mathrm{C}$. Además, se observan grandes poros redondeados en los lugares donde se encontraban inicialmente las partículas de cobre o de bronce. Estos fenómenos se ven acrecentados al sinterizar a la temperatura más elevada (Figs. 9 y 10, correspondientes al acero 304L con $15 \%$ de bronce sinterizado a $1.250{ }^{\circ} \mathrm{C}$ y al acero $316 \mathrm{~L}$ con $20 \%$ de cobre sinterizado a $1.150{ }^{\circ} \mathrm{C}$, respectivamente).

Por tanto, se aprecia cómo para contenidos bajos de aleante, la sinterización que tiene lugar es en

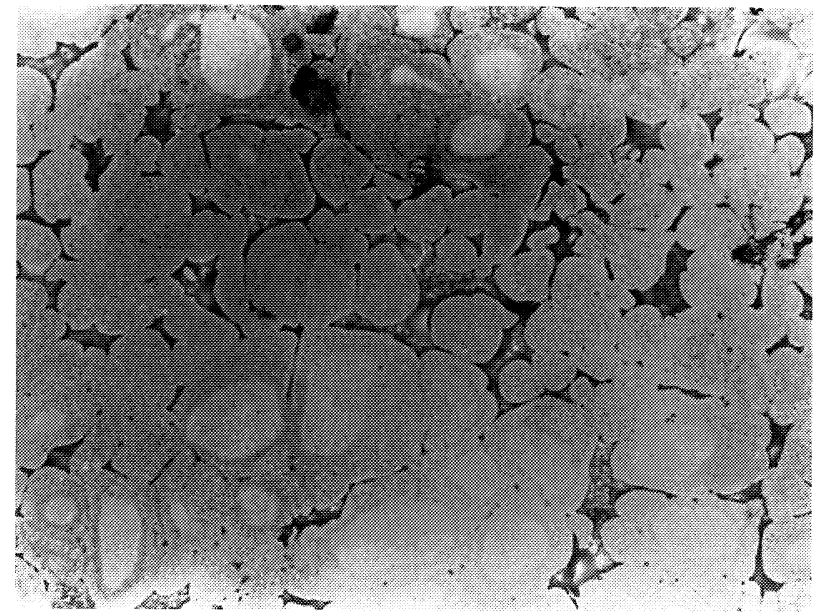

FIG. 10.- Microestructura del acero inoxidable $316 \mathrm{~L}$ aleado con $20 \%$ de cobre sinterizado a 1.150

${ }^{\circ} \mathrm{C}$. Ataque: $\mathrm{HCl}+\mathrm{HNO}_{3}+$ etanol. $\times 125$.

FIG. 10.- Microstructure of $316 \mathrm{~L}$ stainless steel alloyed with $20 \%$ copper sintered at $1,150{ }^{\circ} \mathrm{C}$. Etching: $\mathrm{HCl}+\mathrm{HNO}_{3}+$ ethanol. $\times 125$.

fase líquida transitoria, mientras que para contenidos superiores es en fase líquida permanente.

\section{ANÁLISIS DE RESULTADOS}

Como se puede observar en la figura 1, la densidad en verde de los aceros inoxidables mezclados con cobre y con bronce aumenta con el contenido de aleante empleado. Esto es, la compresibilidad de los aceros inoxidables mejora, como era de esperar, con el contenido de aleante, incluso considerando que tanto el cobre como el bronce tienen mayor densidad que el acero inoxidable. Además, se observa que la compresibilidad es mayor en el caso del cobre, al ser el polvo de bronce más duro y, por tanto, menos compresible que el de cobre.

En la figura 2 se aprecia que la densidad de los aceros sinterizados aumenta con el contenido de cobre o de bronce, para ambas temperaturas de sinterización. Esto se debe a dos causas fundamentales: a la mayor densidad del cobre y del bronce con respecto al acero inoxidable, que tiene influencia sobre todo al emplear altos contenidos de aleante, y a que el cobre y el bronce provocan la aparición de fase líquida que activa el proceso de sinterización.

En lo que respecta a la variación dimensional, se observa (Fig. 3) que el cobre provoca un hinchamiento del acero hasta contenidos aproximados del $15 \%$ para ambas temperaturas de sinterización, contrayendo para el $20 \% \mathrm{Cu}$. En el caso del bronce, el acero se contrae para todos los contenidos de este aleante al sinterizar a $1.150{ }^{\circ} \mathrm{C}$, mientras que cuando se sinteriza a $1.250{ }^{\circ} \mathrm{C}$ se produce un 
hinchamiento hasta aproximadamente un $10 \%$ de bronce. Esta contracción que se produce para elevados contenidos de cobre o de bronce se debe a fenómenos de reapilamiento similares a los observados en aleaciones $\mathrm{Fe}-\mathrm{Cu}$ y $\mathrm{Fe}-\mathrm{Cu}-\mathrm{C}$ sinterizados (11). En la figura 9 se aprecian con claridad los fenómenos de coalescencia y reapilamiento típicos de la sinterización en fase líquida.

En la figura 4 se observa la influencia de la temperatura de sinterización y del aleante añadido sobre la resistencia a la tracción del acero inoxidable 316L. Se advierte que para bajos contenidos, tanto de cobre como de bronce ( 4 y $8 \%$ ), la resistencia a la tracción del acero inoxidable prácticamente no mejora, mientras que para los contenidos más elevados esta propiedad acusa un fuerte incremento. Además, se puede observar que la resistencia a la tracción alcanza valores más altos para la aleación con bronce que con cobre, lo que se debe al mayor endurecimiento que produce el bronce, como ya se puso de manifiesto en los aceros inoxidables infiltrados. También se obtienen mejores resultados absolutos para los aceros sinterizados a $1.150{ }^{\circ} \mathrm{C}$ que a $1.250{ }^{\circ} \mathrm{C}$; esto, unido a la mayor resistencia a la tracción del acero $316 \mathrm{~L}$ sinterizado a $1.250{ }^{\circ} \mathrm{C}$ frente al sinterizado a $1.150^{\circ} \mathrm{C}$, da lugar a que la sinterización a baja temperatura sea más efectiva desde el punto de vista mecánico. Esto puede ser debido, además, a que a $1.250{ }^{\circ} \mathrm{C}$ se puede producir una cierta volatilización del cobre o del estaño, más acusada en valores elevados de elemento de adición.

La ductilidad de los aceros inoxidables $316 \mathrm{~L}$ y 304L (Fig. 5) no se ve sustancialmente afectada al ser aleados con bronce, excepto en el caso del acero $304 \mathrm{~L}$ aleado con $20 \%$ para ambas temperaturas de sinterización.

Es de resaltar que, en aceros sinterizados con cobre (Fe-C-Cu-P), se ha observado que para contenidos de cobre superiores al $5 \%$ aparece cobre libre (11 y 12). Normalmente, y pese a que la solubilidad del cobre en el hierro es aproximadamente el $9 \%$, en la mayoría de los aceros sinterizados la presencia de carbono o fósforo hacen que a partir del 5 ó 6 $\%$ aparezca cobre libre en la microestructura.

\section{CONCLUSIONES}

En los aceros aleados con cobre y con bronce, lo primero a destacar es un aumento de densidad cuando se sinterizan con ambos a las dos temperaturas de sinterización estudiadas. Cabe resaltar que el cobre y el bronce provocan la aparición de la fase líquida que activa el proceso de sinterización. Así, la resistencia a la tracción del acero inoxidable, prácticamente no mejora para bajos contenidos tanto de cobre como de bronce (4 y $8 \%$, en los que se producen procesos de sinterización en fase líquida transitoria), mientras que para contenidos más elevados de aleante (15 y $20 \%$, con sinterización en fase líquida permanente) esta propiedad acusa un fuerte incremento. Además, la resistencia a la tracción alcanza valores más elevados en la aleación con bronce que con cobre, debido al mayor endurecimiento que produce el primero, como se puso de manifiesto en los aceros inoxidables infiltrados. También se debe destacar que la ductilidad no se ve sustancialmente afectada cuando estos aceros se alean con bronce.

\section{Agradecimiento}

Los autores quieren agradecer a la CICYT su apoyo a través del proyecto MAT-94-0230.

\section{REFERENCIAS}

(1) Reinshagen, J.H. y Neupaver, A.J. Ad. Powder Metall., 2, 1989: 283-296.

(2) Lei, G.H. y German, R.M. Mod. Dev. Powder Metall., 16, 1985: 261

(3) Itzhak, D. y Aghion, E. Corros. Sci., 23, 1983: 1.0851.094.

(4) Ahlberg, E., Engdahl, P. y Johansson, R. World Conf. in Powder Metallurgy. EPMA \& Institute of Metals. Londres, 1, 1990: 419-432.

(5) Reinshagen, J.H. y Mason, R.P. Ad. Powder Metall., 5, 1992: 385-397.

(6) Feddrizzi, L., Molinari, A., Deflorian, F., Tiziani, A. y BONORA, P.L. World Conf. in Powder Metallurgy. EPMA \& Institute of Metals. Londres. 2, 1990: 319.

(7) Chattarjee, S.K., Warwick, M.E. y Maykuth, D.J. Mod. Dev. Powder Metall., 16, 1985: 277.

(8) Ro, D.H. y Klar, E. Mod. Dev. Powder Metall., 13, 1981: 247.

(9) Velasco, F., Ibars, J.R., Ruiz Roman, J.M., Torralba, J.M. y Ruiz Prieto, J.M. Corrosion, 52 (1), 1996: 47-52.

(10) Proyecto CICYT:MAT-0980-C03-02

(11) Cambronero, L.E.G., Torralba, J.M. y Ruiz Prieto, J.M. Science of Sintering. Plenum Press. 1989: 487-494.

(12) Tabeshfar, K. y Chadwick, G.A. Powder Metall., 27 (1), 1984: 19-24. 